\title{
Clinical effect on uncontrolled asthma using a novel digital automated self-management solution: a physician-blinded randomised controlled crossover trial
}

\author{
Henrik Ljungberg ${ }^{1,2,3}$, Anna Carleborg ${ }^{2,3}$, Hilmar Gerber ${ }^{4}$, \\ Christina Öfverström ${ }^{4}$, Jakob Wolodarski ${ }^{4}$, Faiza Menshi ${ }^{2,5}$, Mikaela Engdahl $^{2,5}$, \\ Marianne Eduards ${ }^{6}$ and Björn Nordlund (10) ${ }^{1,2,3}$
}

Affiliations: 'Lung-Allergy Dept, Astrid Lindgren Children's Hospital, Karolinska University Hospital, Stockholm, Sweden. 'Dept of Women's and Children's Health, Karolinska Institutet, Stockholm, Sweden. ${ }^{3}$ MediTuner AB, Stockholm, Sweden. ${ }^{4}$ Sophiahemmet Health Care Centre, Stockholm, Sweden. ${ }^{5}$ Children and Adolescents Health Care Dept in Huddinge, Astrid Lindgren Children's Hospital, Karolinska University Hospital, Stockholm, Sweden. 'Liljeholmen Health Care Centre, Stockholm Health Care Services (SLSO), Stockholm County Council, Stockholm, Sweden.

Correspondence: Björn Nordlund, Dept of Asthma and Allergy Research, QB84, Karolinska University Hospital, Karolinska vägen 37A, 17176 Stockholm, Sweden. E-mail: bjorn.nordlundaki.se

@ERSpublications

AsthmaTuner is an automated electronic clinical decision support system that improves symptom control in patients with uncontrolled asthma http://bit.ly/2MhsNAT

Cite this article as: Ljungberg $\mathrm{H}$, Carleborg $\mathrm{A}$, Gerber $\mathrm{H}$, et al. Clinical effect on uncontrolled asthma using a novel digital automated self-management solution: a physician-blinded randomised controlled crossover trial. Eur Respir J 2019; 54: 1900983 [https://doi.org/10.1183/13993003.00983-2019].

\section{ABSTRACT}

Introduction: AsthmaTuner is a novel self-management system consisting of a patient app, a cloud-based storage solution and a healthcare interface. Patients use Bluetooth spirometers to measure lung function (forced expiratory volume in $1 \mathrm{~s}$ ) and can register symptoms. They then receive immediate feedback on asthma control and an image of the correct inhaler(s) to use and the dose. The aim of this pilot study was to evaluate the effect of AsthmaTuner on symptom control and adherence compared with conventional treatment.

Material and methods: This multicentre physician-blinded crossover trial randomised patients in two groups that started with 8 weeks of AsthmaTuner or conventional treatment using a personalised printed treatment plan, with 2 weeks of washout between the crossover treatments. Participants in a primary or paediatric care setting in Sweden with asthma diagnosis, uncontrolled symptoms and Asthma Control Test (ACT) score $<20$ points were included. Symptom control was analysed using t-tests for the difference between the group means of the sums of ACT scores at each treatment end-visit, with 95\% confidence intervals. Medical Adherence Report Scale (MARS) scores captured differences in adherence (remembering to take asthma medication) between treatment periods.

Results: The study population consisted of 77 patients ( $60 \%$ females). The ACT score significantly improved with AsthmaTuner compared with conventional treatment (mean ACT difference 0.70, 95\% CI 0.06-1.34; $\mathrm{p}=0.03$ ). Adherence did not improve significantly in all participants, but did improve among those in primary care who used AsthmaTuner an average of once a week or more compared with conventional treatment (mean MARS difference $0.45,95 \%$ CI 0.13-0.77; $\mathrm{p}=0.01$ ).

Conclusions: AsthmaTuner improved symptom control in patients with uncontrolled asthma compared with conventional treatment. 


\section{Introduction}

Current asthma management comprises excellent medications and guidelines, but many patients remain symptomatic [1], suggesting that management regimens are not optimised or not available for all patients. A further cause of symptoms is suboptimal adherence to prescribed asthma treatment, reported in $>50 \%$ of all individuals with asthma [2]. Reasons for nonadherence range from conscious decisions to ignore asthma treatments to misunderstanding instructions or forgetting to take the medication.

Digital solutions can encourage greater patient involvement in self-management, thereby improving asthma. Simple and easy digital solutions are promising strategies providing patient and clinical decision support, but they should be developed with patient input and tested for efficacy in patient symptom control and adherence. AsthmaTuner is a cloud-based eHealth solution with a healthcare interface and patient app that enables self-monitoring of asthma symptoms and lung function with a Bluetooth spirometer. The app provides patients with an automated treatment recommendation based on the characteristics of symptom control according to the Global Initiative for Asthma (GINA) [3]. The idea for AsthmaTuner originated from meta-analyses showing that patient education in self-management and self-monitoring of lung function and symptoms, coupled with adjustable treatment plans, is more effective compared with other forms of asthma self-management [4].

The aim of this randomised blinded controlled crossover pilot study was to compare AsthmaTuner with a conventional treatment using an individual printed treatment plan in terms of symptom control and remembering to take prescribed asthma medication.

\section{Material and methods \\ Study design}

This multicentre randomised controlled physician-blinded crossover pilot trial consisted of two groups who initially received 8 weeks of either AsthmaTuner or conventional treatment, with a washout period of 2-4 weeks between treatments (figure 1). The primary outcome of symptom control was assessed with the Asthma Control Test (ACT) or Childhood Asthma Control Test (C-ACT) at baseline and at the end-visit in each treatment period. The secondary outcome of adherence was assessed by the Medical Adherence Report Scale (MARS) based on how often respondents forgot to take asthma medications. The randomisation was done for each participant at each site by a study nurse opening a sealed envelope with the study identifier indicating the order of treatment, either AsthmaTuner first and conventional treatment last or the reverse order. The physician was blinded for the randomisation, and individual treatment plans were prescribed for both AsthmaTuner and conventional treatments at study enrolment. Participating centres were endorsed to comply with Swedish national guidelines for the treatment and management of asthma [5], which closely resemble GINA guidelines [3]. The regional board of the Ethical Committee in Stockholm (2015/1527-31/1 and 2016/1546-32) and the Swedish Medical Products Agency (5.1-2016-19829) approved the study. The study was registered at ClinicalTrials.gov with identifier number NCT02571309.

\section{Intervention}

AsthmaTuner (MediTuner, Stockholm, Sweden) is a certified (CE-marked) cloud computing-based system with a healthcare interface and a downloadable patient app (Android or iOS). AsthmaTuner was conceptualised and developed in response to the perceived gap between guidelines/treatment recommendations and clinical practice. The primary aims were to facilitate distribution of treatment plans to patients and to improve self-management and education for patients, as the software helps patients decide the current state of their asthma. Recently, the term electronic clinical decision support system (eCDSS) has gained acceptance [6, 7]. It is important to achieve a high degree of acceptability to clinicians and patients by focusing on automation, data presentation and design, as well as alignment with professional workflow. To achieve the best results, AsthmaTuner was developed in collaboration with the

This article has supplementary material available from erj.ersjournals.com

This study is registered at ClinicalTrials.gov with identifier number NCT02571309.

Data sharing: All collected individual participant data are available and after de-identification data that underline the results reported in this article will be shared. Study protocol and statistical analysis plan, informed consent, clinical study report, and data set with analytical code will be available immediately after publication. Data will be available for investigators whose proposed use of the data has been approved by an independent review committee identified for this purpose and for any type of analyses. Proposal may be submitted up to 36 months following article publication. To gain data access, data requestors should be directed to B. Nordlund and will need to sign a data access agreement. All data are stored for 10 years at Karolinska Institutet (Stockholm, Sweden).

Received: 15 May 2019 | Accepted after revision: 8 Aug 2019 


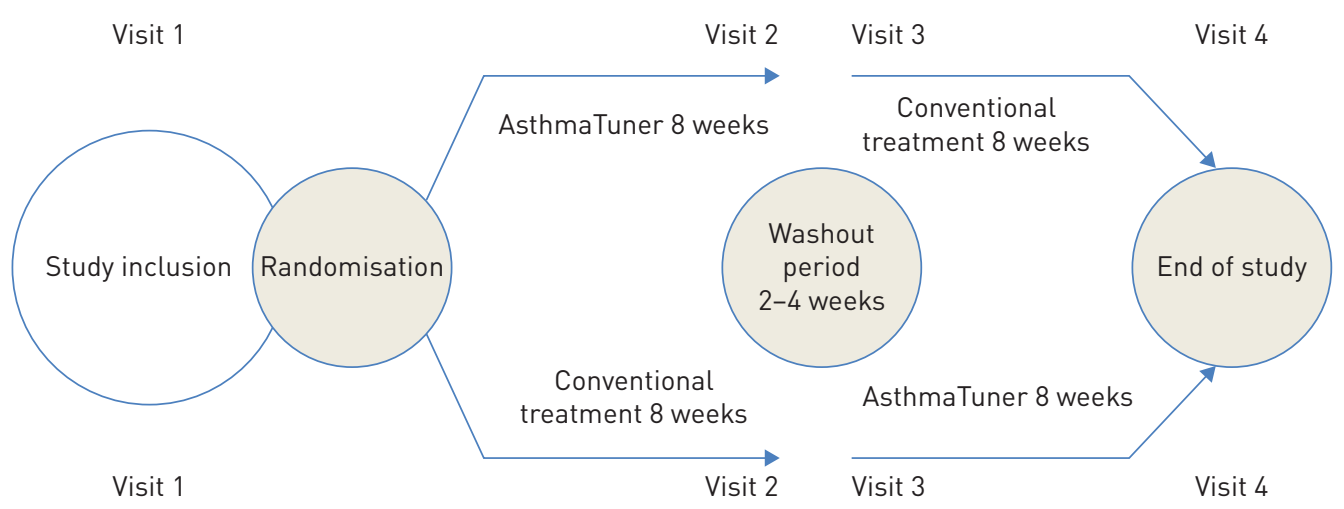

FIGURE 1 Study design of the multicentre randomised controlled physician-blinded crossover pilot trial assessing the effect of treatment with AsthmaTuner on the Asthma Control Test compared with conventional treatment using a personalised printed treatment plan.

Swedish Asthma and Allergy Association patient organisation and patients through focus groups, and with clinicians through interviews with doctors and nurses from public healthcare, as well as private practitioners and online clinics. The intended use of AsthmaTuner is to automate asthma self-management by letting patients register symptoms and measure forced expiratory volume in $1 \mathrm{~s}\left(\mathrm{FEV}_{1}\right)$ with a Bluetooth spirometer (MIR SmartOne; Medical International Research, Rome, Italy) (figure 2). The patient then receives immediate feedback on the status of symptom control (controlled, partly controlled or uncontrolled) and a treatment recommendation, with an image of the correct inhaler or other type of medication and the dose. Symptom control is quantified based on lung function (percentage of personal best $\mathrm{FEV}_{1}$, using a cut-off $\leqslant 80 \%$ ) and symptoms during the last week, based on four questions identifying: 1) need for rescue medication more than twice due to asthma symptoms, 2) any daytime symptoms, 3) nocturnal symptoms/awakenings and 4) limitation in physical activities [3]. Supplementary table S1 shows the algorithm classifying patient symptom control in AsthmaTuner. AsthmaTuner offers patients and healthcare providers longitudinal data views of assessed symptom control, prescribed treatments and lung function measurements. The back-end data storage of the cloud-based system provides information about participant adherence with AsthmaTuner use.

\section{Conventional treatment}

Conventional treatment was defined as nondigital self-management using individual printed treatment plans, which contained treatment adjustments of prescribed medications according to symptoms of controlled, partly controlled or uncontrolled asthma, along with instructions according to national guidelines [5].

\section{Subjects}

The study subjects were children aged $\geqslant 6$ years and adults with at least a doctor's diagnosis of asthma, and ACT/C-ACT scores $<20$ points from May 2016 to September 2018. Exclusion criteria were presence of any comorbidity with significant impact on symptom control, participation in drug trials and patient/caregiver difficulties in reading Swedish. The study was conducted in Stockholm, Sweden, in the primary healthcare sector and specialised paediatric healthcare, at Liljeholmen Health Care Centre, Sophiahemmet Health Care Centre and Astrid Lindgren Children's Hospital. Supplementary figure S1 shows the total number of randomised patients and dropouts. The study population was all randomised participants with complete ACT/C-ACT information who fulfilled all four visits.

\section{Inhalation technique}

Participants' inhaler use was assessed at the baseline visit, and if the demonstration was unsuccessful according to criteria for dry powder and metered dose inhalers [8], they were trained by a study nurse to use it correctly.

\section{Questionnaires}

Patients or caregivers completed a structured health questionnaire at the first visit, providing information on demographics, asthma, comorbidities and treatment. Symptom control was assessed at baseline and at end-visit in each treatment period with a validated ACT in patients aged $\geqslant 12$ years [9] and C-ACT in children aged $6-11$ years [10]. A mean score $\leqslant 19$ points indicated uncontrolled asthma in both tests. The 


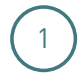

Asthma medications/ adjust treatment
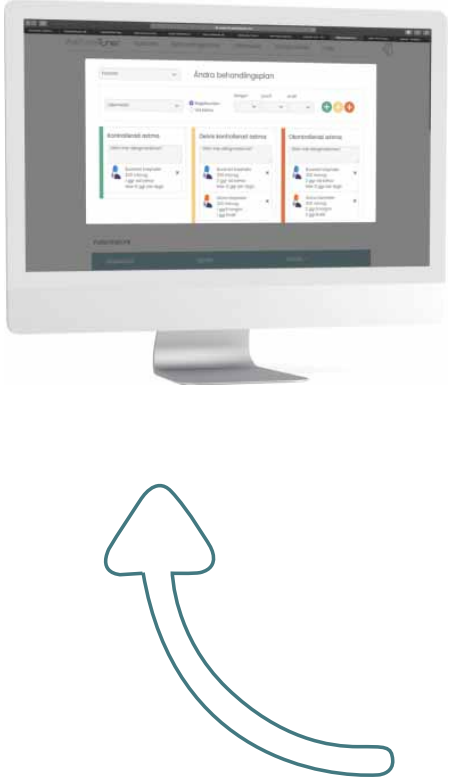

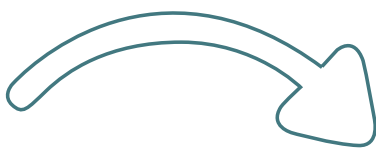

2

Automated treatment

plan based on lung

function and symptoms

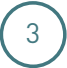

Assess asthma control

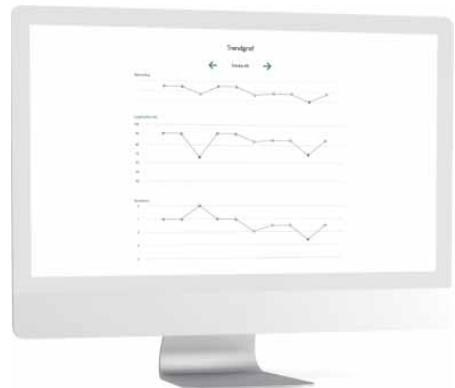

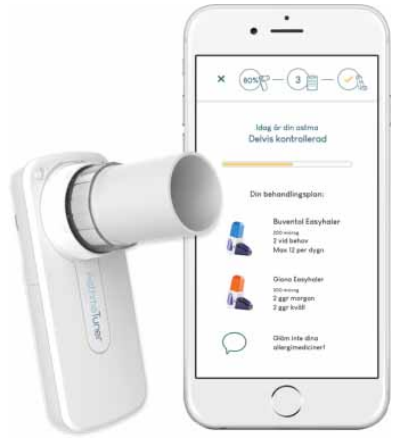

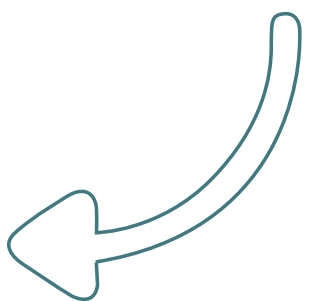

FIGURE 2 AsthmaTuner is a certified (CE-marked) electronic clinical decision support system with cloud-based data storage, a healthcare interface and a downloadable patient app (Android or iOS). The intended use of AsthmaTuner is to automate asthma self-management by letting healthcare providers prescribe treatment and patients register symptoms and measure forced expiratory volume in $1 \mathrm{~s}$ with a Bluetooth spirometer. The patient then receives immediate feedback on asthma control and an image of the correct inhaler(s) to use and the dose. The healthcare provider can simultaneously monitor the patient's asthma control.

MARS medication adherence score captured how often responders forgot to take their asthma medication ( $1=$ always, $2=$ often, $3=$ sometimes, $4=$ rarely and $5=$ never) at baseline and at end-visit for each period.

\section{Lung function}

Dynamic spirometry measurements (forced vital capacity (FVC) \% predicted, $\mathrm{FEV}_{1} \%$ pred, $\mathrm{FEV}_{1} / \mathrm{FVC}$ and forced expiratory flow at $50 \%$ of $\mathrm{FVC}\left(\mathrm{FEF}_{50 \%}\right) \%$ pred) were performed in accordance with guidelines using Hedenström/Soleymar or Zapletal reference values [11].

\section{Data management}

The clinical research organisation Karolinska Trial Alliance (Stockholm, Sweden) conducted the data review, supervised the study according to Good Clinical Practice standards and conducted structured data reviews at each study centre.

\section{Statistical analyses}

The sample size was estimated assuming that AsthmaTuner would improve the average ACT/C-ACT score by 2 points compared with conventional treatment (mean SD 3.3). Assuming a dropout rate up to $10 \%$, our power calculation estimated that enrolment of 43 adults and 43 children would be clinically relevant and feasible to attain $80 \%$ power at a $5 \%$ significance level. The interventional effect of AsthmaTuner on 
ACT/C-ACT scores was compared with conventional treatment according to intention-to-treat analyses, including all patients $(\mathrm{n}=77)$ assessed with complete information on ACT/C-ACT from each visit. All analyses were stratified by primary healthcare and paediatric specialist care setting.

The distribution of ACT/C-ACT scores at end-visits 2 and 4 was checked using histograms and quantilequantile plots, and indicated no large deviation from the normal distribution. Examination of the ACT/ C-ACT scores revealed that the baseline measurements of period 2 were close to the outcome measurements of period 1, indicating that the baseline measurements of period 2 are not comparable between the randomised groups. Consequently, statistical testing of the carryover effect may be biased, as the carryover effect was observed even when no or negligible carryover effect was present. For this reason, and in line with FLEISS [12], only the ACT/C-ACT measurements at the end-visits were used in the statistical analyses in which carryover and treatment effects were evaluated. Differences in crossover effects were tested using t-tests for the difference between the group means of the sums of ACT/C-ACT scores at visits 2 and 4. Differences in treatment effects were tested using t-tests for the difference between the group means of the differences of ACT/C-ACT scores at visits 2 and 4 . When adjusting for adherence and care facility, linear regression was used, with the randomisation group as the explanatory variable of interest, adding the number of assessments with AsthmaTuner as the natural logarithm, and an indicator for care facility as additional adjustment variables. The estimate and confidence interval for the randomisation group variable was divided by 2 in order to get the estimate for the difference between AsthmaTuner and conventional treatment. Sequence effects on ACT/C-ACT scores between period 1 and 2 were estimated using the t-test. Differences in the MARS question about respondents' forgetfulness to take their asthma medication showed comparable baseline measurements between period 1 and 2; hence, the differences in MARS scores between end-visit and baseline were used as outcomes in the analyses. All t-tests were applied using the formulae given in section 10.1.2 in FLEISS [12]. p-values $<0.05$ were considered significant. R version 3.5.0 (www.r-project.org) was used for all statistical analyses.

\section{Results}

In total, 77 out of 89 (87\%) randomised participants completed the study: 37 patients from primary healthcare and 40 schoolchildren from paediatric specialist care settings (supplementary figure S1). Some participants were lost to follow-up $(n=6)$, withdrew their study consent because of technical problems with their mobile phone $(n=3)$ or terminated study participation for medical conditions other than asthma $(\mathrm{n}=3$ : severe snake bite reaction, pertussis and another respiratory diagnosis). No severe device deficiencies were observed, but insufficient connection between the Bluetooth spirometer and Android phones was reported, as was the inability to use the app after mistaken installation onto a tablet device.

Table 1 shows the baseline characteristics of the study population; table 2 shows stratification by primary care $(n=37)$ and schoolchildren in paediatric specialist care $(n=40)$. The mean \pm sD age was $22 \pm 14.5$ years for the study population: median 33.1 years for the primary care cohort and median 11.7 years for the paediatric cohort. The mean daily doses of inhaled corticosteroids (ICSs) prescribed in the individual treatment plans for either AsthmaTuner or conventional treatment were $450 \mu \mathrm{g}$ (budesonide or equivalent) for controlled asthma, $762 \mu \mathrm{g}$ for partly controlled asthma and $956 \mu \mathrm{g}$ for uncontrolled asthma (table 1). Allergic comorbidities of eczema, rhino-conjunctivitis and food allergy were prevalent in $27 \%, 41 \%$ and $35 \%$ of the study population, respectively. The number of AsthmaTuner assessments during the treatment period was significantly higher in the paediatric cohort than in the primary care cohort (mean 37.6 versus 17.6; $\mathrm{p}=0.006$ ). Supplementary figure S2 demonstrates proportions of participants with uncontrolled, partly controlled and controlled asthma as defined by the AsthmaTuner algorithm across study weeks 1-9. The proportion of participants with uncontrolled asthma decreased from $37 \%$ to $8 \%$ between week 1 and 9 . The average number of assessments and automated treatment changes by AsthmaTuner at each study week are described in supplementary table S2.

Table 3 shows the effect size of AsthmaTuner compared with a conventional treatment with printed treatment plans on ACT/C-ACT scores. The ACT/C-ACT end-visit scores were significantly better with AsthmaTuner than with conventional treatment (mean difference $0.70,95 \%$ CI $0.06-1.34 ; \mathrm{p}=0.03$ ) (figure 3a). However, AsthmaTuner showed no statistically significant effect on ACT/C-ACT scores, stratified by primary care cohort, compared with conventional treatment (figure $3 \mathrm{~b}$ ). The paediatric specialist care cohort alone showed significantly improved ACT/C-ACT scores with AsthmaTuner compared with conventional treatment (mean difference 0.97, 95\% CI 0.13-1.81; $\mathrm{p}=0.02$ ) (figure 3c). Tests of differences in crossover effects were all far from significant ( $p$-values $0.63,0.49$ and 0.23 ) for all participants and when stratified by primary care or paediatric specialist care cohort.

Linear regression in table 4 estimates crude and adjusted effects on ACT/C-ACT scores between AsthmaTuner and conventional treatment. No additional effect of AsthmaTuner on ACT/C-ACT scores was found when adjusting for either number of assessments with AsthmaTuner or care facility (primary or 
TABLE 1 Characteristics of the study population at study baseline

\begin{tabular}{|c|c|c|c|}
\hline & Subjects & Median (IQR; range) & Mean士sd \\
\hline Age years & 77 & $16.0(12-30 ; 6-72)$ & $22.0 \pm 14.5$ \\
\hline ACT/C-ACT score & 77 & $16.0(13.8-18.0 ; 8-19)$ & $15.6 \pm 3.1$ \\
\hline MARS score & 77 & $4.0(3.0-5.0 ; 2-5)$ & $3.6 \pm 0.9$ \\
\hline FVC \% pred & 75 & $88.0(80.0-98.0 ; 60.0-118.0)$ & $88.9 \pm 12.4$ \\
\hline FEV $_{1} \%$ pred & 76 & $85.3(76.0-96.9 ; 55.0-137.0)$ & $86.4 \pm 14.2$ \\
\hline $\mathrm{FEV}_{1} / \mathrm{FVC}$ & 77 & $0.84(0.78-0.87 ; 0.52-0.98)$ & $0.82 \pm 0.09$ \\
\hline \multicolumn{3}{|l|}{ Prescribed treatment plan } & $74.6 \pm 22.3$ \\
\hline Controlled asthma ICS $\mu \mathrm{g}$ & 77 & $400(320-640 ; 0-1472)$ & $450 \pm 259$ \\
\hline Partly controlled asthma ICS $\mu \mathrm{g}$ & 77 & $800(480-960 ; 0-1600)$ & $762 \pm 386$ \\
\hline Uncontrolled asthma ICS $\mu \mathrm{g}$ & 77 & $960(750-1200 ; 0-2080)$ & $956 \pm 423$ \\
\hline Assessments with AsthmaTuner & 77 & $18.5(11-34 ; 1-217)$ & $28.0 \pm 32.5$ \\
\hline Female & $46(59.7 \%)$ & & \\
\hline ICS & $74(96.1 \%)$ & & \\
\hline Montelukast & $32(41.6 \%)$ & & \\
\hline Long-acting $\boldsymbol{\beta}_{2}$-agonist & $72(93.5 \%)$ & & \\
\hline \multicolumn{4}{|l|}{ Allergic self-reported comorbidities } \\
\hline Eczema & $21(27.3 \%)$ & & \\
\hline Rhino-conjunctivitis & $32(41.6 \%)$ & & \\
\hline Food allergy & $27(35.1 \%)$ & & \\
\hline Randomisation AsthmaTuner first & $38(49.4 \%)$ & & \\
\hline
\end{tabular}

IQR: interquartile range; range: minimum-maximum; ACT: Asthma Control Test; C-ACT: Childhood Asthma Control Test; MARS: Medical Adherence Report Scale (how often respondents forgot to take asthma medications: 1=always, 2=often, $3=$ sometimes, 4=rarely and 5=never); FVC: forced vital capacity; FEV : forced expiratory volume in $1 \mathrm{~s} ; \mathrm{FEF}_{50 \%}$ : forced expiratory flow at $50 \%$ of FVC; ICS: inhaled corticosteroid (daily dose of budesonide or equivalent).

TABLE 2 Characteristics of study participants stratified by primary care and paediatric specialist care at study baseline

\begin{tabular}{|c|c|c|c|c|c|c|}
\hline & \multicolumn{3}{|c|}{ Primary care } & \multicolumn{3}{|c|}{ Paediatric specialist care } \\
\hline & Subjects & Median (IQR; range) & Mean士sd & Subjects & Median (IQR; range) & Mean士sD \\
\hline Age years & 37 & $32.0(23.0-40.0 ; 13-72)$ & $33.1 \pm 13.7$ & 40 & $12.5(9-14 ; 6-17)$ & $11.7 \pm 3.2$ \\
\hline MARS score & 37 & $4.0(3.0-4.0 ; 2.0-5.0)$ & $3.6 \pm 0.8$ & 40 & $4.0(3.0-5.0 ; 2.0-5.0)$ & $3.7 \pm 1.0$ \\
\hline FVC \% pred & 36 & $85.0(78.0-93.6 ; 60.0-109.0)$ & $84.6 \pm 11.0$ & 39 & $94.0(82.0-103.0 ; 71.0-118.0)$ & $93.0 \pm 12.3$ \\
\hline $\mathrm{FEV}_{1} \%$ pred & 37 & $83.7(75.5-94.9 ; 55.0-115.3)$ & $84.2 \pm 12.8$ & 39 & $89.0(76.0-100 ; 62.0-137)$ & $88.5 \pm 15.3$ \\
\hline Controlled asthma ICS $\mu \mathrm{g}$ & 37 & 320 (400-710; 0-1472) & $537 \pm 285$ & 40 & 320 (267-500; 0-800) & $370 \pm 205$ \\
\hline Partly controlled asthma ICS $\mu \mathrm{g}$ & 37 & $800(800-1240 ; 0-1600)$ & $923 \pm 436$ & 40 & $640(400-800 ; 0-1280)$ & $612 \pm 258$ \\
\hline Uncontrolled asthma ICS $\mu \mathrm{g}$ & 37 & $1200(800-1600 ; 0-2080)$ & $969 \pm 498$ & 40 & $800(640-960 ; 0-1280)$ & $791 \pm 249$ \\
\hline Assessments with AsthmaTuner & 37 & $15.0(9.5-24 ; 1.0-46.0)$ & $17.6 \pm 11.1$ & 40 & $26.0(13.0-48.3 ; 4.0-217)$ & $37.7 \pm 42.0$ \\
\hline Female & $28(75.7 \%)$ & & & $18(45 \%)$ & & \\
\hline Eczema & $9(24.3 \%)$ & & & $12(30 \%)$ & & \\
\hline Rhino-conjunctivitis & $17(45.9 \%)$ & & & $15(37.5 \%)$ & & \\
\hline Food allergy & $11(29.7 \%)$ & & & $16(40 \%)$ & & \\
\hline Randomisation AsthmaTuner first & $16(43.2 \%)$ & & & $23(57.5 \%)$ & & \\
\hline
\end{tabular}

IQR: interquartile range; range: minimum-maximum; ACT: Asthma Control Test; C-ACT: Childhood Asthma Control Test; MARS: Medical Adherence Report Scale (how often respondents forgot to take asthma medications: 1=always, 2=often, 3=sometimes, 4=rarely and 5=never); FVC: forced vital capacity; $\mathrm{FEV}_{1}$ : forced expiratory volume in $1 \mathrm{~s} ; \mathrm{FEF}_{50 \%}$ : forced expiratory flow at $50 \%$ of FVC; ICS: inhaled corticosteroid (daily dose of budesonide or equivalent). 
TABLE 3 Effect on the Asthma Control Test (ACT)/Childhood Asthma Control Test (C-ACT) scores at end-visit after treatment with AsthmaTuner or conventional treatment in all participants, and stratified by primary care and paediatric specialist care cohort

Subjects
ACT/C-ACT score at end-visit:

AsthmaTuner

\begin{tabular}{lllcr}
\hline All participants & 77 & $19.45(18.70-20.21)$ & $18.75(17.97-19.53)$ & $0.70(0.06-1.34) ; p=0.03$ \\
Primary care & 37 & $19.14(18.08-20.19)$ & $18.78(17.63-19.94)$ & $0.33(-0.68-1.35) ; p=0.51$ \\
Paediatric specialist care & 40 & $19.75(18.65-20.85)$ & $18.73(17.61-19.84)$ & $0.97(0.13-1.81) ; p=0.02$
\end{tabular}

\section{ACT/C-ACT score at end-visit:} conventional treatment
ACT/C-ACT difference: AsthmaTuner versus conventional treatment
$0.70(0.06-1.34) ; p=0.03$

$0.33(-0.68-1.35) ; p=0.51$

$0.97(0.13-1.81) ; p=0.02$

Data are presented as mean $(95 \% \mathrm{Cl})$, unless otherwise stated. Differences in crossover effect between study period 1 and 2 were nonsignificant in all participants $(p=0.62)$, primary care $(p=0.49)$ and paediatric care $(0.23)$.

paediatric care), or both, compared with crude analysis. Significant sequence effects on ACT/C-ACT scores for both AsthmaTuner and conventional treatment were observed between period 1 and 2 (AsthmaTuner: period 1 versus period 2 , mean \pm SD $4.28 \pm 3.70$ versus $0.67 \pm 2.09$; $\mathrm{p}<0.001$; conventional treatment: mean \pm SD $2.61 \pm 3.25$ versus $-0.08 \pm 2.80 ; \mathrm{p}<0.001)$.

Participant adherence to remember to take asthma medication was assessed with the MARS questionnaire (table 5). Adherence was not improved with AsthmaTuner compared with conventional treatment. However, adherence was increased in 27 participants using AsthmaTuner once a week or more, based on derived data from the cloud-based back-end, compared with conventional treatment in the primary care setting (mean MARS difference 0.45 , 95\% CI $0.13-0.77$; $\mathrm{p}=0.01$ ). No similar effect was detected in 35
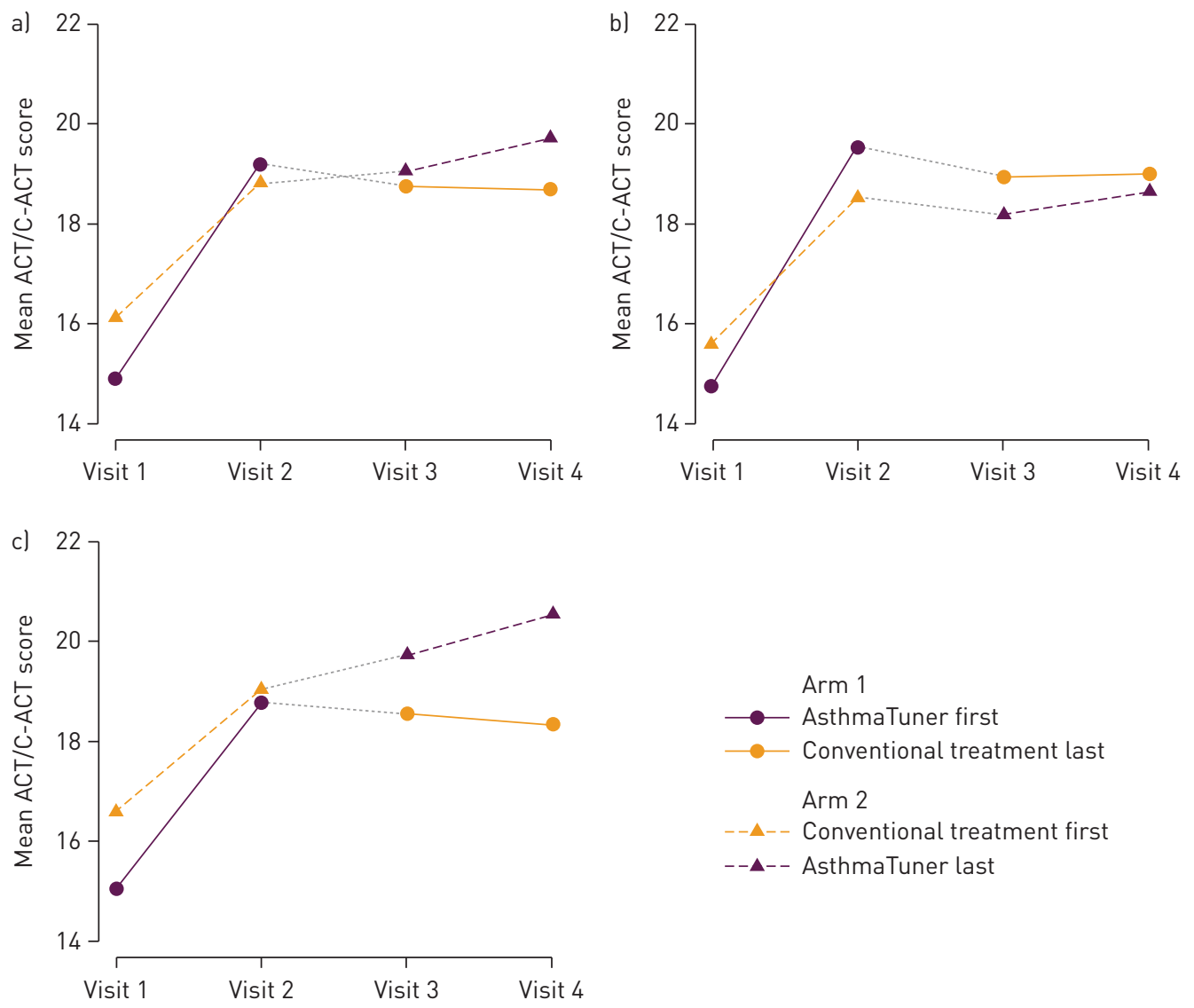

Arm 1

$\longrightarrow$ AsthmaTuner first

- Conventional treatment last

Arm 2

$--\Delta-$ Conventional treatment first

--1- - AsthmaTuner last

FIGURE 3 Mean Asthma Control Test (ACT)/Childhood Asthma Control Test (C-ACT) scores at baseline and end-visits after AsthmaTuner or conventional treatment in a) all participants $(n=77)$, b) the primary care cohort $(n=37)$ and $c)$ the paediatric specialist care cohort $(n=40)$. 
TABLE 4 Linear regression estimating the effect on the Asthma Control Test (ACT)/Childhood Asthma Control Test (C-ACT) scores between AsthmaTuner and conventional treatment adjusted for number of assessments with AsthmaTuner and primary or paediatric care facility

\begin{tabular}{lc} 
& $\begin{array}{c}\text { Difference in ACT/C-ACT scores } \\
\text { between AsthmaTuner and } \\
\text { conventional treatment }\end{array}$ \\
\hline Unadjusted & $0.70(0.06-1.34) ; p=0.03$ \\
Adjusted for number of AsthmaTuner assessments (log) & $0.70(0.03-1.36) ; p=0.04$ \\
Adjusted for care facility (primary or paediatric) & $0.66(0.02-1.31) ; p=0.04$ \\
Adjusted for AsthmaTuner assessments (log) and care facility & $0.62(-0.05-1.30) ; p=0.07$
\end{tabular}

Data are presented as $\beta$-coefficient $(95 \% \mathrm{CI})$, unless otherwise stated. ${ }^{\#}: \mathrm{n}=77$.

schoolchildren that used AsthmaTuner once a week or more in the paediatric cohort. The p-values for the tests of differences in crossover effects were all far from significant ( $p$-values $0.37,0.40$ and 0.65 ), for both cohorts, the primary care cohort and the paediatric specialist care cohort, respectively; hence, differences in carryover effects were ruled out.

\begin{abstract}
Discussion
This crossover pilot randomised controlled trial showed significantly better symptom scores of ACT/ C-ACT with AsthmaTuner than with conventional treatment using a printed personalised treatment plan. Our assessment of the effect on adherence, i.e. remembering to take asthma medication, in the overall study population revealed no significant effect compared with conventional treatment. However, adherence was enhanced in 27 participants of the primary care cohort that used AsthmaTuner an average of once a week or more compared with conventional treatment. Overall, the novel digital AsthmaTuner system seems to be an easy and effective strategy for managing uncontrolled asthma.

In this intention-to-treat study, the primary outcome of validated ACT/C-ACT score was significantly improved (mean increase 0.7 points) in patients with uncontrolled asthma using AsthmaTuner compared with conventional treatment. The effect on ACT/C-ACT was most prominent in schoolchildren of the paediatric specialist care cohort. The findings imply that AsthmaTuner can be recommended for patients with uncontrolled asthma. Empowering patients by linking self-monitored data to an adjustable individual treatment plan is a promising strategy for self-management; however, only a few studies are available and most are small in scale with conflicting evidence. The randomised controlled trial by RYAN et al. [13] on 288 adults and children with poorly controlled asthma found no significant effect on symptom control comparing mobile phone-based transmission of symptoms, medication and feedback prompting a patient action plan versus printed self-management. However, symptom control did improve significantly after 6 months in both study arms. The randomised controlled trial by PERRY et al. [14] on 34 adolescents with
\end{abstract}

TABLE 5 Effect on Medical Adherence Report Scale (MARS)" , difference in participants' forgetfulness to take their asthma medication, within and between treatment with AsthmaTuner or conventional treatment in all study participants, and stratified by cohorts in primary care, paediatric specialist care and at least weekly average use of AsthmaTuner

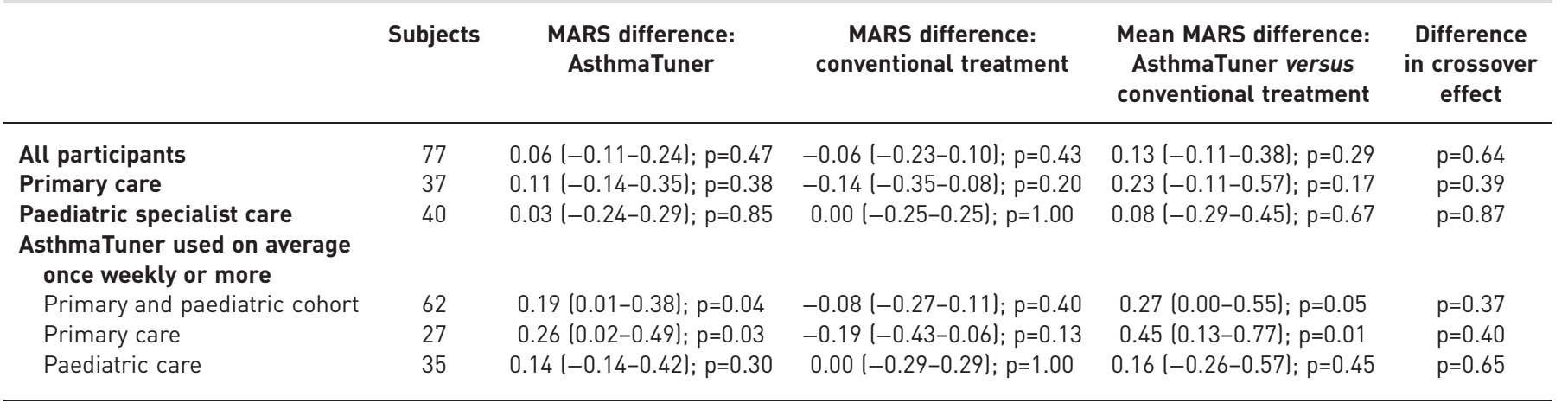

Data are presented as mean $\left(95 \% \mathrm{CI}\right.$ ), unless otherwise stated. ${ }^{\#}$ : MARS (how often respondents forgot to take asthma medications): $1=$ always, 2=often, $3=$ sometimes, $4=$ rarely, $5=$ never. 
persistent asthma found no significant effect on ACT scores using a smartphone versus print-based treatment plans. However, BURBANK et al. [15] found a significant improvement in symptom control in 20 adolescents in one of the arms in their study, suggesting that personalised treatments with mobile-based asthma action plans are feasible for communicating individual treatments and improving symptom control. These trials have functions related to web-based systems where patients can simply access and submit their data. Having access to a treatment plan on a web-based system, instead of a traditional printed treatment plan, does not seem to improve asthma control. Instead, it seems that an adjustable treatment plan stored on a smartphone allows for more effective control. In our study, almost all participants were prescribed ICS control medication at each symptom control level and this could also explain some of the differences in efficacy in symptom control. Ryan et al. [13] demonstrated that ICS was first prescribed at step 2 of a total of four treatment steps, while neither PERRY et al. [14] nor BuRBANK et al. [15] clearly indicated the ICS level or dose given. The AsthmaTuner system has the advantage of a minimum need for manual input. This may contribute to ease of use and acceptance (e.g. there was no need for manual input from the wireless spirometer). Also, the result with a clear presentation of treatment recommendation as an image of the inhaler/medicine and number of inhalations required may help the user.

Assessment of different digital solutions requires an understanding of how the targeted patient groups perceive and use a specific digital tool. In this study, the number of assessments with AsthmaTuner was significantly higher in the paediatric cohort than in the primary care cohort, indicating that schoolchildren were motivated to use AsthmaTuner more frequently. Patient involvement with the first available prototype of AsthmaTuner was established by in-depth interviews of patients and children's caregivers. These interviews gave important feedback on the functionality and usability of AsthmaTuner, and on whether testing pulmonary function with wireless spirometers to assess possible bronchial obstruction encouraged patients to take control of their self-management. The interviews also clarified that answering symptom questions on a regular basis was tiring and that automated feedback on asthma status was more desirable. The historical view of collected data, shared with the healthcare provider, was an appreciated reminder of previous health status, and aided healthcare providers in monitoring and prioritising patients in most need of care. The AsthmaTuner system is currently being implemented in European countries.

Overall, AsthmaTuner did not show a clear benefit on reminding participants to take their medication over conventional treatment. Stratified analysis of participants using AsthmaTuner at least once a week or more indicated improved adherence in the primary care cohort. Studies using an electronic monitoring device with an audio-visual reminder showed significant improvements in adherence to ICS in school-aged children [16] and in adults with asthma [17]. The evaluated version of AsthmaTuner lacked electronic monitoring devices with audio-visual reminders, but an upgraded, currently available version of AsthmaTuner has settings for self-monitoring and medication reminders. One limitation of our study is our failure to assess adherence in caregivers to children because they typically supervise medication.

\section{Strengths and limitations}

Regarding the generalisability of this study, the multicentre design and the inclusion of participants from two different settings (i.e. primary healthcare and paediatric specialist care) is a strength. Most asthma cases in Sweden are managed in these two settings. ARAújo et al. [18] successfully assessed the clinical feasibility of web-based versus standard asthma self-management in a small crossover study. The crossover randomised controlled trial design has strengths by adding sensitivity and power when compared with parallel arms [19], and reduces the influence of confounding factors because each crossover participant serves as their own control. The four follow-up visits in our study are in line with Swedish guidelines that recommend at least two follow-up visits per year for managing uncontrolled asthma. The use of a printed personalised treatment plan in the conventional treatment group is promoted in Swedish national asthma programmes [5]; nevertheless, few patients receive treatment plans and that is an evidence-based care gap [20].

There are reports of improved learned behaviour using internet-based asthma self-management solutions $[21,22]$. A possible limitation of this study is its crossover design, which carries an obvious risk that the effect of the first period treatment is carried over into the next period. The use of AsthmaTuner may lead to behavioural changes that are difficult to extinguish with a washout period between treatment periods. However, in this study, we saw no significant difference in ACT/C-ACT scores between management periods 1 and 2, indicating that our imposed washout period prevented crossover effects and that differences in carryover effects could be ruled out. Instead, we saw that the baseline measurements of period 2 were close to the outcome measurements of period 1, indicating that the baseline measurements of period 2 are not comparable between the randomised groups. Consequently, the statistical analysis of the carryover effect may be biased, as the carryover effect could be seen even when no or a negligible carryover effect exists. We overcame this problem, in line with FLEISS [12], by using only the ACT/C-ACT 
measurements at the end-visits in the statistical analyses where carryover and treatment effects were evaluated. Furthermore, significant sequence effects of period 1 were observed for both AsthmaTuner and conventional treatment on uncontrolled asthma, which underlines how effective improved self-management is on symptom control [23] and that AsthmaTuner is a digital modification of evidence-based conventional treatment.

Asthma diagnosis needs to be regularly verified in both adults and children [24]. A limitation of this real-life study is the criterion used of a doctor's asthma diagnosis. We did not confirm participants asthma objectively before enrolment. However, a great potential of an eCDSS is providing patients with wireless spirometry and healthcare information on lung function to objectively verify the diagnosis based on variability and treatment response.

The scope for future studies will be to assess AsthmaTuner in a larger population with unselected symptom control. The main outcomes will be evaluation of the effects on symptom control and adherence, as well as characterisation of specific phenotypes based on time-serial measurements of lung function in relation to symptoms and exacerbations. The goal will be to develop mathematical algorithms for generating individual prescribed action plans to prevent exacerbations.

\section{Conclusions}

This study indicates significant improvements in symptom scores of ACT/C-ACT in patients with uncontrolled asthma using AsthmaTuner compared with conventional treatment with printed personalised treatment plans. The effect on adherence to remember to take asthma medication was not significantly improved in the overall study population compared with conventional treatment. Adherence was only improved in participants of the primary care cohort that used AsthmaTuner on average once a week or more compared with conventional treatment. We conclude that AsthmaTuner, a novel digital self-management system, is a simple and easy solution that can be recommended for the management of uncontrolled asthma in schoolchildren and adults.

Acknowledgements: We acknowledge all the study participants and all the staff involved in this study at Liljeholmen Health Care Centre, Sophiahemmet Health Care Centre and Astrid Lindgren Children's Hospital at Karolinska University Hospital (all Stockholm, Sweden).

Conflict of interest: H. Ljungberg is a co-founder and holds shares in the company MediTuner that owns AsthmaTuner. A. Carleborg reports personal fees from MediTuner, outside the submitted work; she is an employee of MediTuner and has warrants in the company. H. Gerber has nothing to disclose. C. Öfverström has nothing to disclose. J. Wolodarski has nothing to disclose. F. Menshi has nothing to disclose. M. Engdahl has nothing to disclose. M. Eduards has nothing to disclose. B. Nordlund is a co-founder and holds shares in the company MediTuner that owns AsthmaTuner.

Support statement: The study was supported by the Bio-X programme of Uppsala BIO, Stockholm County Council Innovation Foundation, Capio Research Foundation, Sweden's Innovation Agency (Vinnova) and MediTuner AB. Funding information for this article has been deposited with the Crossref Funder Registry.

\section{References}

1 Demoly P, Paggiaro P, Plaza V, et al. Prevalence of asthma control among adults in France, Germany, Italy, Spain and the UK. Eur Respir Rev 2009; 18: 105-112.

2 Chung KF, Wenzel SE, Brozek JL, et al. International ERS/ATS guidelines on definition, evaluation and treatment of severe asthma. Eur Respir J 2014; 43: 343-373.

3 Bateman ED, Hurd SS, Barnes PJ, et al. Global strategy for asthma management and prevention: GINA executive summary. Eur Respir J 2008; 31: 143-178.

4 Gibson PG, Powell H, Coughlan J, et al. Self-management education and regular practitioner review for adults with asthma. Cochrane Database Syst Rev 2003; 1: CD001117.

5 National Board of Health and Welfare of Sweden. Nationella riktlinjer för vård vid astma och kroniskt obstruktiv lungsjukdom (KOL). [National guidelines for the treatment of asthma and chronic obstructive pulmonary disease (COPD).] 2017. www.socialstyrelsen.se/nationellariktlinjerastmaochkol Date last accessed: August 18, 2019.

6 Matui P, Wyatt JC, Pinnock H, et al. Computer decision support systems for asthma: a systematic review. NPJ Prim Care Respir Med 2014; 24: 14005.

7 Courbis AL, Murray RB, Arnavielhe S, et al. Electronic clinical decision support system for allergic rhinitis management: MASK e-CDSS. Clin Exp Allergy 2018; 48: 1640-1653.

8 Aksu F, Sahin AD, Sengezer T, et al. Effect of training by a physician on dynamics of the use of inhaler devices to improve technique in patients with obstructive lung diseases. Allergy Asthma Proc 2016; 37: 98-102.

9 Nathan RA, Sorkness CA, Kosinski M, et al. Development of the asthma control test: a survey for assessing asthma control. J Allergy Clin Immunol 2004; 113: 59-65.

10 Liu AH, Zeiger R, Sorkness C, et al. Development and cross-sectional validation of the Childhood Asthma Control Test. J Allergy Clin Immunol 2007; 119: 817-825.

11 Miller MR, Hankinson J, Brusasco V, et al. Standardisation of spirometry. Eur Respir J 2005; 26: 319-338.

12 Fleiss JL. The Design and Analysis of Clinical Experiments. New York, Wiley, 1999.

13 Ryan D, Price D, Musgrave SD, et al. Clinical and cost effectiveness of mobile phone supported self monitoring of asthma: multicentre randomised controlled trial. BMJ 2012; 344: e1756. 
14 Perry TT, Marshall A, Berlinski A, et al. Smartphone-based vs paper-based asthma action plans for adolescents. Ann Allergy Asthma Immunol 2017; 118: 298-303.

15 Burbank AJ, Lewis SD, Hewes M, et al. Mobile-based asthma action plans for adolescents. J Asthma 2015; 52: 583-586.

16 Chan AH, Stewart AW, Harrison J, et al. The effect of an electronic monitoring device with audiovisual reminder function on adherence to inhaled corticosteroids and school attendance in children with asthma: a randomised controlled trial. Lancet Respir Med 2015; 3: 210-219.

17 Charles T, Quinn D, Weatherall $\mathrm{M}$, et al. An audiovisual reminder function improves adherence with inhaled corticosteroid therapy in asthma. J Allergy Clin Immunol 2007; 119: 811-816.

18 Araújo L, Jacinto T, Moreira A, et al. Clinical efficacy of web-based versus standard asthma self-management. J Investig Allergol Clin Immunol 2012; 22: 28-34.

19 Cleophas TJ, de Vogel EM. Crossover studies are a better format for comparing equivalent treatments than parallel-group studies. Pharm World Sci 1998; 20: 113-117.

20 To T, Guttmann A, Lougheed MD, et al. Evidence-based performance indicators of primary care for asthma: a modified RAND Appropriateness Method. Int J Qual Health Care 2010; 22: 476-485.

21 van der Meer V, Bakker MJ, van den Hout WB, et al. Internet-based self-management plus education compared with usual care in asthma: a randomized trial. Ann Intern Med 2009; 151: 110-120.

22 van Gaalen JL, Beerthuizen T, van der Meer V, et al. Long-term outcomes of internet-based self-management support in adults with asthma: randomized controlled trial. J Med Internet Res 2013; 15: e188.

23 Guevara JP, Wolf FM, Grum CM, et al. Effects of educational interventions for self management of asthma in children and adolescents: systematic review and meta-analysis. BMJ 2003; 326: 1308-1309.

24 Aaron SD, Vandemheen KL, FitzGerald JM, et al. Reevaluation of diagnosis in adults with physician-diagnosed asthma. JAMA 2017; 317: 269-279. 